\title{
Impact of clonal growth on effective population size in Hymenoxys herbacea (Asteraceae)
}

\author{
LG Campbell ${ }^{1}$ and BC Husband \\ Department of Botany, University of Guelph, Guelph, Ontario, Canada N1G 2W1
}

\begin{abstract}
By influencing the proliferation of different genotypes, clonal growth can affect the maintenance of genetic variability and magnitude of genetic drift within plant populations. However, estimates of effective population size rarely incorporate the contribution of both asexual and sexual reproduction. We estimated effective size $\left(N_{\mathrm{e}}\right)$ for two populations of the clonal, self-incompatible plant, Hymenoxys herbacea, using a stagestructured demographic model for organisms with asexual and sexual recruitment and then examined the impact of reproductive strategy using an elasticity analysis. Plant rosettes monitored in two successive years had high survival rates in both populations (mean 0.94). The mean number of sexually derived recruits per initial ramet was 0.041 (SE
\end{abstract}

0.039), whereas the mean number of clonal recruits was 0.61 (SE 0.90). Effective size was 1642 and 5769 in the two populations and the $N_{\mathrm{e}} / N$ ratio averaged 0.34 , comparable to values for other clonal species. Elasticity analysis indicated that increases in both clonal and sexual recruitment cause an increase in $N_{\mathrm{e}}$ while increasing the variance reduced $N_{\mathrm{e}}$. However, $N_{\mathrm{e}}$ was more sensitive to changes in the mean and variance of asexual recruitment than sexual recruitment. These results highlight the importance of considering asexual modes of reproduction when examining the role of genetic stochasticity in populations.

Heredity (2005) 94, 526-532. doi:10.1038/sj.hdy.6800653

Published online 2 March 2005

Keywords: asexual reproduction; demography; elasticity analysis; genetic drift; Lakeside Daisy; self-incompatible

\section{Introduction}

Plant reproductive systems have diverse genetic consequences for populations, particularly as a result of variation in the balance of asexual and sexual modes of propagation. Asexual reproduction via clonal propagation is widespread in flowering plants as a result of lateral expansion through corms, bulbils, bulbs, rhizomes and stolons (Jackson et al, 1985; van Groenendael and de Kroon, 1990). By influencing the representation of alleles and genotypes in subsequent years, this mode of reproduction may influence effective population size $\left(N_{\mathrm{e}}\right)$ and genetic diversity in contradictory ways. By enhancing the variance in genet size and reducing opportunities for recombination, differential clonal reproduction may reduce effective population size and consequently enhance the magnitude of drift (Orive, 1993; Milgroom, 1996). On the other hand, clonal expansion may decrease the rate at which alleles are lost, enhance heterozygosity and thereby increase effective population size (Balloux et al, 2003; Bengtsson, 2003). This ambiguity is mirrored by empirical studies of genetic diversity in partially asexual organisms (Ellstrand and Roose, 1987; Gabrielsen and Brochmann, 1998). However, there are no empirical investigations of the impact of clonality on $N_{\mathrm{e}}$ in natural populations (although see Yonezawa, 1997).

Correspondence: BC Husband, Department of Botany, University of Guelph, Guelph, Ontario, Canada N1G 2W1.

E-mail: bhusband@uoguelph.ca

${ }^{1}$ Current address: Department of Evolution, Ecology and Organismal Biology, Ohio State University, 1735 Neil Avenue, Columbus, OH, USA. Received 3 September 2004; accepted 12 January 2005; published online 2 March 2005
Empirical studies of $N_{\mathrm{e}}$ in clonal organisms have been limited by the lack of estimators that incorporate both sexual and asexual reproductive modes. Commonly, effective size is estimated prospectively as the census number $(N)$ of a population adjusted for the demographic characteristics that deviate from an ideal population, such as nonrandom mating, uneven sex ratios and fluctuations in population size over time (Wright, 1969; Hall et al, 1996). This approach is based on classical theory of effective population size (Wright, 1931 and summarized in Crow, 1954; Kimura and Crow, 1963) pertaining to sexual, monoecious species with nonoverlapping generations. More recently, age-specific, overlapping-generation models have been developed, but these are not applicable when plant age is not known or difficult to assess (Hill, 1972, 1979; Emigh and Pollak, 1979). These models have recently been extended to stage-structured populations with mixed asexual and sexual reproductive systems and overlapping generations (Orive, 1993; Yonezawa, 1997; Yonezawa et al, 2000); however, they have rarely been applied to clonal plant populations and have never been used to evaluate the relative sensitivity of $N_{\mathrm{e}}$ to clonal versus sexual reproduction.

Hymenoxys herbacea (EL Greene) Cusick (Asteraceae) (Cusick, 1991) is endemic to the Great Lakes region of Canada and the USA and is currently known from only 40 locations (Campbell et al, 2001). Populations occur in open alvar and cliff habitats, which are variable in size and isolated from each other by forest habitat. The resulting geographic structure may enhance genetic drift and the loss of genetic diversity in this species. On the other hand, loss of alleles may be tempered by the presence of clonal growth via rhizomes. Understanding 
effective size and its determinants may have ecological as well as evolutionary implications because this plant is governed by a sporophytic self-incompatibility system (De Mauro, 1993), which ensures that successful fertilization occurs only between genotypes carrying different self-incompatibility (SI) alleles (de Nettancourt, 1977). Small effective population sizes may be sufficient to cause the loss of SI alleles and thereby affect seed production and ultimately population viability. In this study, we estimated effective population size $\left(N_{\mathrm{e}}\right)$ in two $H$. herbacea populations using a stage-structured demographic model that incorporates the magnitude and variance of clonal as well as sexual reproduction (Yonezawa, 1997; Yonezawa et al, 2000). We asked the following specific questions: (1) what is the value of $N_{\mathrm{e}}$ and $N_{\mathrm{e}} / N$ ratio in natural populations of $H$. herbacea; (2) which demographic attributes of $H$. herbacea have the greatest impact on $N_{e}$; and (3) What is the relative impact of clonal versus sexual recruitment on values of $N_{\mathrm{e}}$ ?

\section{Materials and methods}

\section{Study organism and field sites}

$H$. herbacea plants comprise one or more rosettes (up to $10 \mathrm{~cm}$ in height), each with a cluster of fleshy, strappedshaped leaves. In any given year, plants may expand clonally through rhizomatous growth to produce additional rosettes (De Mauro, 1989; De Mauro, 1993) or reproduce sexually to produce seeds. Solitary inflorescences of yellow ray (female) and disc (hermaphrodite) florets are produced in spring (May through June) and are insect pollinated (De Mauro, 1993). Seeds are produced approximately 3 weeks after pollination (Campbell, 2001) and are dispersed primarily by gravity (De Mauro, 1993). As there is no enforced seed dormancy and seeds germinate immediately when the soil is warm and moist (De Mauro, 1993), or not at all, the contribution of the seed bank to population growth is expected to be minimal.

Effective size was estimated in two replicate populations (CPL and HL) from the Bruce Peninsula, Ontario, Canada. These were chosen randomly from the existing populations that are accessible and least vulnerable to human impact.

\section{Effective population size model}

We estimated effective population size $\left(N_{\mathrm{e}}\right)$ and the $N_{\mathrm{e}} / N$ ratio for each population using a stage-structured model developed for populations with mixed sexual and asexual reproduction and overlapping generations (Yonezawa, 1997). Following Yonezawa (1997), $N_{\mathrm{e}}$ was estimated as:

$$
N_{\mathrm{e}}=\frac{1}{L} \frac{2 N}{2(1+\alpha)\left(\bar{u}-\bar{u}^{2}\right)+(1-\bar{u})(S+A \delta)}
$$

where $N$ is the number of ramets (ie, rosettes) in the population; $\alpha$ is the deviation from random outcrossing and is derived as $\beta /(2-\beta)$, where $\beta$ is the population selfing rate; $\bar{u}$ is the total fraction of individuals surviving to the next year $\left(=D_{1} u_{.1}+D_{2} u_{.2}+D_{3} u_{.3}\right)$, where $D_{i}$ represents the proportion of individuals within the population that belong to the demographic stages $i=1$, 2,3 and $u_{x}$ is the probability of a plant in stage $x$ surviving 1 year. Variable $\bar{u}^{2}$ is calculated as $D_{1}\left(u_{.1}\right)^{2}+$
$D_{2}\left(u_{.2}\right)^{2}+D_{3}\left(u_{.3}\right)^{2}$ and $S$ as $(1-\alpha)+(1+\alpha)\left(V_{k} / k\right)$, where $k$ and $V_{k}$ are the average and variance of the number of sexual recruits per ramet. Variable A is calculated as 2(1 $+\alpha)\left(V_{c} / c\right)-S$, where $c$ and $V_{c}$ are the average and variance of the number of asexual recruits per ramet; $\delta$ is the proportion of new recruits that were asexually derived; and $L$ is the generation length, defined as the mean age at which new plants produce offspring (asexual or sexual) (Yonezawa, 1997). Below we describe the methods used for estimating each of these demographic parameters.

\section{Estimates of model parameters}

Total number of ramets: Census population size $(N)$ was estimated by conducting a complete census of rosettes within each of the two populations at the beginning of May 2000. In addition, the number of reproductive rosettes $\left(N_{\mathrm{r}}\right)$ was estimated in a complete census during a single visit to both populations in the first week of June 1999 and 2000.

Survival rates and transition probabilities: Five plots $(30 \mathrm{~cm} \times 30 \mathrm{~cm})$ were randomly positioned throughout both populations. Each of 150 rosettes within the plots were tagged and numbered in early June 1999 and then classified into one of three demographic stages:

(1) Juvenile-less than 1" tall, 4-6 leaves, not flowering;

(2) Non-sexual adult-more than 1" tall, more than 6 leaves, not flowering;

(3) Reproductive adult-inflorescence present or evidence of flowering within the current flowering season.

Seed and seedling stages were not monitored since they would have either died or grown into the above categories by the June census. In June 2000, we relocated the marked rosettes and scored them again as present/ absent and, if present, according to one of the three stageclasses. From this 2-year survey, we calculated: (1) the proportion of plants in each stage-class $\left(D_{1}, D_{2}, D_{3}\right)$ each year; (2) the proportion of plants initially in each stageclass that survived 1 year $\left(u_{1}, u_{2}, u_{3}\right)$, and (3) the probabilities of transition among stages $\left(u_{i j} ; i=\right.$ stage 1,2 , 3 in 1999 and $j=$ stage 1, 2, 3 in 2000). Parameters $\bar{u}$ and $\bar{u}^{2}$ were then estimated as described above.

Recruitment rates: To estimate the number of sexual and asexual recruits, five additional plots $(30 \mathrm{~cm} \times 30 \mathrm{~cm})$ were randomly positioned in each population in June 1999 and all rosettes within these plots were tagged and numbered for a total of 150 . In June 2000, we relocated the tagged rosettes and also counted the number of new rosettes within the plots. Any new rosette that was physically connected to a tagged rosette was classified as a product of vegetative growth and thus an asexual recruit. Any new rosette that was not connected to a tagged rosette was classified as a sexual recruit. From this information, we calculated the mean $(c)$ and variance $\left(V_{c}\right)$ of the number of asexual recruits per rosette and the mean $(k)$ and variance $\left(V_{k}\right)$ of the number of sexual recruits per rosette. The calculation of $V_{c}$ was straightforward as each asexual recruit was traceable to a parent rosette. This was not the case for sexual recruits. However, seeds of $H$. herbacea disperse by gravity (De Mauro, 1993), and thus we assigned the closest flowering rosette as the putative parent. Because the sexual recruits 
were sparse, no parent rosette was ever assigned more than one recruit. The frequency of asexual recruitment $(\delta)$ was calculated as the number of asexual recruits divided by the total number of new recruits in the plots. Our measure of recruitment assumes that most viable seeds produced by tagged plants remain within the plots, or any that are lost to emigration are balanced by immigration.

Generation length: Generation length $(L)$ was calculated following Yonezawa et al (2000), as:

$$
L=\frac{\sum_{x=1}^{500} \sum_{j=1}^{3} x F_{j} u_{j 1 x}}{\sum_{x=1}^{500} \sum_{j=1}^{3} F_{j} u_{j 1 x}} 24
$$

where $u_{j 1 x}$ is the probability that a newborn will be in stage $j$ at age $x$ and where $x$ spans 500 generations and $F_{j}$ represents the number of new recruits produced either sexually or asexually per plant in stage $j$. Estimates of $u_{j 1 x}$ and $F_{j}$ were generated from the data on stage-specific recruitment and survival described earlier. The estimate is a ratio of age-specific vital rates summed across years and approaches a stable value after 500 years (data not shown).

Population selfing rate: The population selfing rate $(\beta)$ was inferred from the segregation of allozymes among open-pollinated progeny using the mixed mating model of Ritland and Jain (1981). The model estimates the population outcrossing rate $(t)$, which is the mean proportion of offspring per mother plant that are outcrossed. The estimation procedure uses information from three polymorphic loci to generate a multilocus estimate $\left(t_{\mathrm{m}}\right)$ as well as a mean single-locus outcrossing rate $\left(t_{\mathrm{s}}\right)$. The multilocus estimates provide the best measure of true selfing, while the single-locus estimates will also reflect effects of spatial structure and mating with relatives.

To estimate $t_{\mathrm{m}}$ and $t_{\mathrm{s}}$, seed families were collected outside the demographic plots from 25 randomly chosen ramets in each population. From each maternal plant, eight offspring were grown to the juvenile stage in the Botany Department greenhouse, University of Guelph. One young leaf per plant was harvested and ground in $45 \mathrm{ml}$ 'Decodon' extraction buffer (Eckert and Barrett, 1994) after freezing in liquid nitrogen. The homogenate was centrifuged for $10 \mathrm{~min}$ at $4^{\circ} \mathrm{C}$ and the supernatant was applied to a cellulose acetate gel.

An electric current $(200 \mathrm{~V})$ was run across the gel for 45-60 min and a stain consisting of an enzymatic substrate and a colorimetric dye was added to visualize each of three polymorphic enzymes ( $\alpha$-ACP, MDH, PGI). The enzyme acid phosphatase ( $\alpha$-ACP, EC 3.1.3.2.) was resolved on morpholine citrate $(\mathrm{pH}$ 6.5) buffer, while malate dehydrogenase (MDH, EC 1.1.1.37), and phosphoglucose isomerase (PGI, 5.3.1.9.) were resolved on tris-citrate (pH 7.0) buffer systems (Hebert and Beaton, 1989; Manchenko, 1994). Each locus scored in this study (Acp-1, Mdh-1, Pgi-1) consisted of two alleles each, and these were designated ' $f$ ' (fast) or ' $s$ ' (slow) based on their relative mobility.

Outcrossing rates were generated using the maximumlikelihood estimation procedure of Ritland and Jain (1981). Maternal genotypes were inferred from the progeny arrays of genotypes according to the method of Brown and Allard (1970). Maximum-likelihood estimates were found using Newton-Raphson iteration, with standard errors calculated as the SD of 1000 bootstrap values generated with the progeny array as the unit of resampling. Although a number of different initial $t_{\mathrm{m}}$ values were used, $t_{\mathrm{m}}=0.5$ for the HL population and $t_{\mathrm{m}}=0.9$ for the CPL population gave estimates with the highest log-likelihood. The selfing rate was calculated for each population as: $\beta=1-t_{\mathrm{m}}$.

\section{Elasticity Analysis}

Elasticity values describe the proportional change in population $N_{\mathrm{e}}$ resulting from a proportional change in an individual model parameter (de Kroon et al, 2000). For simple linear models, elasticities can be calculated analytically (de Kroon et al, 2000). Because an analytical solution is not readily available for the nonlinear model of $N_{\mathrm{e}}$ used in this study, elasticity was estimated by the average change in $N_{\mathrm{e}}$ as parameter $x$ is increased and decreased by a small percentage, following,

$$
E=\frac{N_{\mathrm{e}(x+x 0.01)}-N_{\mathrm{e}(x-x 0.01)}}{N_{\mathrm{e}} 0.02}
$$

where $N_{\mathrm{e}(x+x 0.01)}$ is the value of $N_{\mathrm{e}}$ calculated after the parameter $x$ is increased (or decreased) by 1\% (Heppell et al, 2000). Elasticity values were calculated for 13 different parameters $\left(N, u_{1}, u_{.2}, u_{.3}, D_{1}, D_{2}, D_{3}, c, V_{c}, k\right.$, $\left.V_{k}, \delta, L, \beta\right)$. All composite variables $\left(A, \alpha, S, \bar{u}, \bar{u}^{2}\right)$, derived from combinations of the above variables, were not included in this analysis.

\section{Results}

\section{Demography and $N_{\mathrm{e}}$ estimates}

The total number of ramets $(N)$ in populations CPL and HL for May 2000 was 6574 and 13756 respectively and the number of reproductive ramets $\left(N_{\mathrm{r}}\right)$, averaged over 2 years, was 118. 5 (SE 49.5) and 435 (SE 139) respectively.

Owing to vandalism, the number of marked plants in the demographic plots was reduced from the initial 150 in 1999 to 148 in population CPL and 138 in population HL in 2000. In 1999, the nonreproductive adult stage was represented by the most rosettes $\left(D_{2}=0.67\right.$ and 0.54 in populations CPL and HL, respectively), followed by reproductive adults $\left(D_{3}=0.24 / 0.30\right)$ and juveniles $\left(D_{1}=0.09 / 0.16\right)$ (Figure 1$)$. Over the 1999-2000 interval, the mean proportion of rosettes surviving was 0.90 for juveniles $\left(u_{.1}\right.$ for population $\mathrm{CPL}=0.85$ and population $\mathrm{HL}=0.95$ )(Figure 1), 0.95 for nonsexual adults (population CPL, $u_{.2}=0.91$; population $\mathrm{HL}, u_{.2}=0.99$ ), and 0.92 for adult plants (population CPL, $u_{.3}=0.94$; population HL, $\left.u_{.3}=0.90\right)$. The survival rate $(u)$ of all marked rosettes was 0.93 in population CPL and 0.96 in population HL. Across the two populations, from 1999 to 2000, an average of $53 \%$ of juvenile individuals remained as juveniles, $32 \%$ became nonsexual adults and four percent became adults (Figure 1, Table 1). Most nonsexual plants (64\%) remained nonsexual. Of all stages, nonsexual individuals were the stage most likely to become reproductive adults (20\%). Reproductive adults in 1999 most often reverted to the nonsexual stage in the following year $(71 \%)$ and rarely remained adult $(6.5 \%)$ (Figure 1$)$. The generation length $(L)$ was 11.3 for population CPL and 21.5 for population HL. 


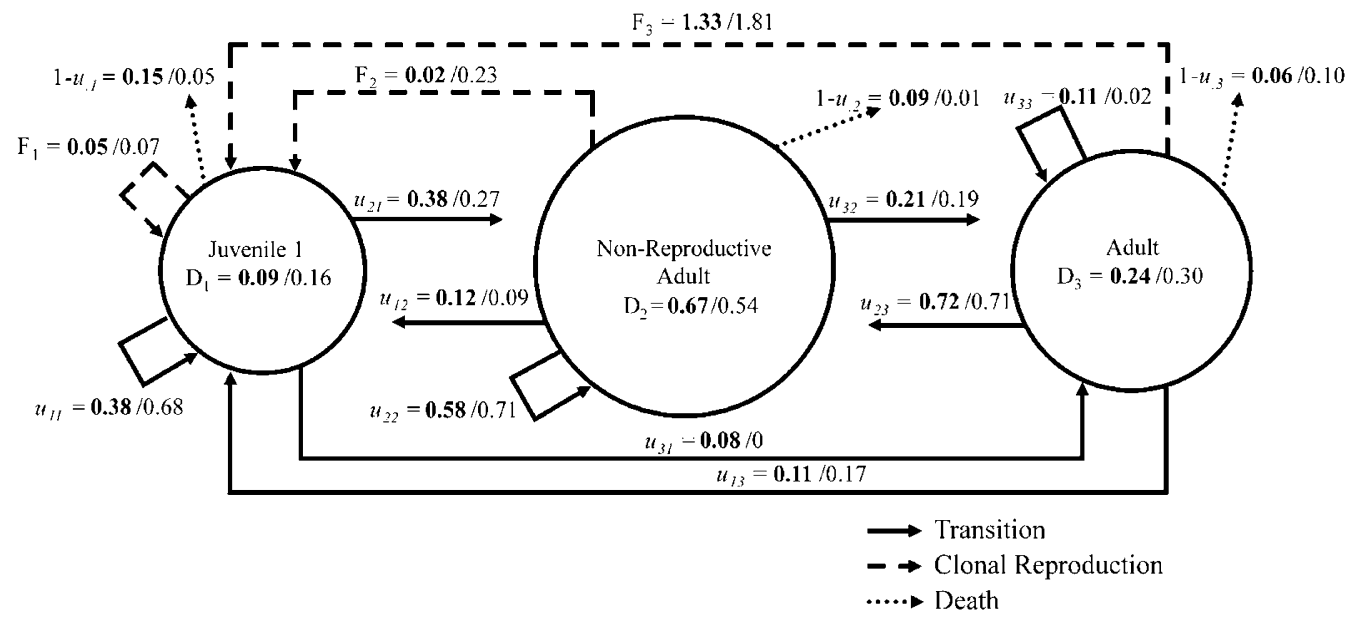

Figure 1 Life-cycle graph of two $H$. herbacea populations (CPL, HL) reflecting the changes in survival and recruitment over a 1-year interval. $F_{i}=$ the number of new recruits produced, either sexually or asexually, per plant in stage $i$; $u_{i j}$ is the probability that a plant in stage $j$ develops to stage $i$ in the next year; $D_{i}$ is the fraction of plants in the demographic stage $i ; 1-u_{. i}$ is the probability that plants of stage $i$ die in the next year. Nomenclature follows that of Yonezawa (1997). Values associated with population CPL are in bold.

Table 1 Stage transitions and mortality over a 1-year interval (1999-2000) for populations CPL and HL of H. herbacea from the Bruce Peninsula, Ontario, Canada

\begin{tabular}{|c|c|c|c|c|c|c|}
\hline \multirow[t]{2}{*}{ Stage in 2000} & \multicolumn{3}{|c|}{ Stage in 1999 (CPL) } & \multicolumn{3}{|c|}{ Stage in 1999 (HL) } \\
\hline & Juvenile & Non-flowering adult & Flowering adult & Juvenile & Non-flowering adult & Flowering adult \\
\hline Juvenile & $5(0.38)$ & $12(0.12)$ & $4(0.11)$ & $15(0.68)$ & $7(0.09)$ & $7(0.17)$ \\
\hline Nonsexual adult & $5(0.38)$ & $57(0.58)$ & $26(0.72)$ & $6(0.27)$ & $53(0.71)$ & $29(0.71)$ \\
\hline Sexual adult & $1(0.08)$ & $21(0.21)$ & $4(0.11)$ & $0(0.00)$ & $14(0.19)$ & $1(0.02)$ \\
\hline Dead & $2(0.15)$ & $9(0.09)$ & $2(0.06)$ & $1(0.05)$ & $1(0.00)$ & $4(0.10)$ \\
\hline
\end{tabular}

Each value represents the number (proportion) of rosettes at a given demographic stage in June 1999 classified as juvenile (4-6 leaves), non-flowering adults ( $>6$ leaves) and flowering adult (reproductive) or absent in 2000.

Within the recruitment plots, 89 new plants were found in population CPL and 84 in population HL, of which $90 \%$ (80 plants) and $97 \%$ (82 plants) were derived clonally, respectively. The mean number of sexually derived recruits per initial ramet, $k$ (variance, $V_{k}$ ), was 0.067 (0.063) in population CPL and 0.015 (0.014) in population HL. The mean (c) number of clonal recruits per ramet (variance, $V_{c}$ ) was $0.62(0.86)$ for population CPL and 0.61 (0.91) for population HL.

Multilocus outcrossing rates (population CPL: $t_{\mathrm{m}} \pm \mathrm{SE}=0.823 \pm 0.137$, population HL: $t_{\mathrm{m}} \pm \mathrm{SE}=0.893 \pm$ 0.100 ) were high and not significantly different from one in either population (population CPL: $t=1.288, n=152$, $P>0.1$; population HL: $t=0.778, n=149, P>0.4)$. In addition, the single-locus outcrossing rates (population CPL: $t_{\mathrm{s}} \pm \mathrm{SE}=0.816 \pm 0.155$, population $\mathrm{HL}: t_{\mathrm{s}} \pm \mathrm{SE}=$ $0.898 \pm 0.115)$ were not significantly different from one (population CPL: $t=1.18, n=152, P>0.2$; population HL: $t=0.884, n=149, P>0.2)$. The selfing rate $(\beta)$ was calculated to be 0.177 for population CPL and 0.107 for population HL.

Using the demographic parameters above and the model of Yonezawa (1997), the effective population size for population CPL was 1642, while $N_{\mathrm{e}}$ for population HL was 5769. The $N_{\mathrm{e}} / N$ ratio was 0.25 for population CPL and 0.42 for population HL (population mean $=0.34$ ).
Elasticity analysis

Consistent across both populations, survival rate of nonsexual adults $\left(u_{.2}\right)$ and the proportion of nonsexual adults $\left(D_{2}\right)$ had the largest and second largest impacts on $N_{\mathrm{e}}$, respectively (Table 2). Both variables affected $N_{\mathrm{e}}$ positively (ie an increase in the variable caused an increase in $N_{\mathrm{e}}$ ). Survival of reproductive adults $\left(u_{.3}\right)$ had the third largest impact on $N_{\mathrm{e}}$ in both populations (Table 2). With respect to reproductive parameters, $N_{e}$ was consistently more sensitive to the variance and mean number of clonal recruits than sexual recruits in both populations. For both reproductive modes, raising mean recruitment increased effective size, whereas increasing the variance reduced effective size.

\section{Discussion}

Our estimates of $N_{\mathrm{e}}$ are comparable to other demographic estimates in plants. Values were similar to the only other estimate of $N_{\mathrm{e}}$ that incorporates asexual and sexual reproduction in a stage-structured model (Yonezawa et al, 2000). Specifically, the $N_{\mathrm{e}}$ of Fritillaria camtschatcensis ranged from 3274 to 5329 in the two populations examined, and the $N_{\mathrm{e}} / N$ ratio was 0.26 . The difference in mean $N_{\mathrm{e}} / N$ ratio between species may be explained, in part, by the lower frequency of nonsexual adults $(3 \%)$ in F. camtschatcensis populations compared to 
Table 2 Elasticity (proportional sensitivity) of effective population size estimates for two populations of $H$. herbacea, CPL and HL, from the Bruce Peninsula, Ontario, Canada

\begin{tabular}{lccc}
\hline Parameter & \multicolumn{2}{c}{ Elasticity values } & Direction \\
\cline { 2 - 3 } & $C P L\left(\times 10^{-3}\right)$ & $H L\left(\times 10^{-3}\right)$ & \\
\hline$N$ & 0.400 & 0.400 & + \\
$u_{.1}$ & 0.187 & 1.392 & + \\
$u_{.2}$ & 1.341 & 5.119 & + \\
$u_{.3}$ & 0.634 & 2.353 & + \\
$D_{1}$ & 0.109 & 0.837 & + \\
$D_{2}$ & 0.784 & 3.032 & + \\
$D_{3}$ & 0.365 & 1.423 & + \\
$C$ & 0.230 & 0.243 & + \\
$V_{c}$ & 0.230 & 0.243 & + \\
$K$ & 0.009 & 0.002 & - \\
$V_{k}$ & 0.009 & 0.002 & - \\
$\delta$ & 0.084 & 0.009 & - \\
$L$ & 0.400 & 0.040 & \\
$\beta$ & 0.015 & 0.015 & \\
\hline
\end{tabular}

Values represent the mean proportional change in $N_{\mathrm{e}}$ as a result of a proportional change (increase and decrease of $1 \%$ ) in the magnitude of each parameter in the estimation model (Heppell et al, 1999). The direction $N_{\mathrm{e}}$ changes as a result of an increase of $1 \%$ is also indicated.

H. herbacea (61\%). From our elasticity analysis, this variable had a relatively large and positive impact on $N_{\mathrm{e}}$. Rates of recruitment through clonal reproduction are also much higher in F. camtschatcensis (5.85 clonal recruits/ramet in $F$. camtschatcensis versus 1.75 in $H$. herbacea), although we would have expected this to increase $N_{\mathrm{e}} / N$ in $F$. camtschatcensis. This incongruence highlights the difficulty of identifying one critical demographic factor, particularly when $N_{\mathrm{e}}$ is the result of interactions among many demographic traits.

A recent review of the literature provides a broader frame of reference for our $N_{\mathrm{e}}$ estimates (Frankham, 1995). From 38 empirical estimates, the $N_{\mathrm{e}} / N$ ratio for demographic studies of plant species averaged 0.32 and ranged from 0.008 to 0.68 . Clearly, the $N_{\mathrm{e}} / N$ ratio of $H$. herbacea (0.34) falls within this range and also within the range predicted by several theoretical models (Nunney, 1991, 1993, 1996).

It is difficult to interpret the significance of clonality for our $N_{\mathrm{e}}$ estimates. In his literature survey, Frankham (1995) did not explore the demographic characters associated with variation in $N_{\mathrm{e}} / N$ ratios. Although effective size estimates for clonal plants do not always incorporate measures of asexual reproduction, $N_{\mathrm{e}} / \mathrm{N}$ ratios from clonal organisms tend to be lower than those published for nonclonal organisms (Orive, 1993; Olfelt et al, 1998; Richman and Kohn, 1999). The generality of this trend is unclear given the small number of studies and the likely presence of confounding life history characters associated with clonality such as perenniality and outcrossing. Moreover, this trend is not consistent with theoretical predictions that suggest clonality will enhance effective size, when clonal reproduction is high or that plants with mixed reproduction modes should be nearly indistinguishable from strictly sexually reproducing plants (Balloux et al, 2003; Bengtsson, 2003).

Elasticity analyses provide the most informative insights into the effects of clonality on $N_{\mathrm{e}}$, while holding other demographic characters constant. Our results suggest that clonality has a greater impact on $N_{\mathrm{e}}$ than sexual reproduction, perhaps because of the higher rate of clonal recruitment in this species. More generally, the results suggest that omitting measures of clonal recruitment from demographic estimates of $N_{\mathrm{e}}$ may bias estimates more than if sexual reproduction was not included. Furthermore, clonality is affecting $N_{\mathrm{e}}$ in different ways. On one hand, higher average clonal recruitment increases $N_{\mathrm{e}}$. The magnitude of this effect is best illustrated by recalculating $N_{\mathrm{e}}$ for our populations using Hill's (1972, Equation 16) model, which accommodates perennial life histories but not asexual reproduction. Under this model, $N_{\mathrm{e}}$ was 298 and 2074, which is less than $1 / 10$ and $1 / 2$ of population CPL and HL, respectively. On the other hand, the variance in asexuality among genets lowers $N_{\mathrm{e}}$. The net effect will therefore depend on the correlation between these two attributes. Indeed, clonality may have little net effect in some species, suggesting there may be no general difference in $N_{\mathrm{e}}$ between clonal and nonclonal plants.

The $N_{\mathrm{e}}$ estimates from our study may be influenced by other demographic factors not taken into consideration, specifically (1) yearly variation in population size and (2) the method of estimation of $N_{\mathrm{e}}$ (ie, demographic versus genetic). Fluctuations in population size may result in very small values of $N_{\mathrm{e}} / N$ and low $N_{\mathrm{e}}$ in animal populations (Vucetich et al, 1997). This has also been shown to be important in plant species such as the tropical aquatic plant, Eichhornia paniculata. Husband and Barrett (1992) found that temporal variation in population size reduced $N_{\mathrm{e}}$ to $50 \%$ of $N$. Vucetich and Waite (1998) illustrated that a minimum of 15 annual counts may be needed to accurately estimate $N_{\mathrm{e}}$. As our estimate for $H$. herbacea is calculated from only 1 year's data, we may have overestimated long-term $N_{\mathrm{e}}$.

Second, it is poorly understood how accurately demographic estimates, based on population structure and vital rates, of $N_{\mathrm{e}}$ predict genetic behaviour of populations. While demographic methods are frequently used and reasonably robust for a wide range of mating systems and dispersal patterns (Heywood, 1986; Basset et al, 2001), few comparisons of $N_{\mathrm{e}}$ involving both demographic and genetic approaches exist (but see Begon et al, 1980; Husband and Barrett, 1992). Husband and Barrett (1992) found that demographic estimates of effective population size were between 2 and 500 times larger than genetic estimates. This again suggests that our $N_{\mathrm{e}}$ values for $H$. herbacea are overestimates and serve as conservative estimates of loss of genetic diversity via drift.

\section{Implications}

How strong is genetic drift in $H$. herbacea? As a rule of thumb, an effective size of at least 5000 individuals is necessary to avoid loss of neutral variation and erosion of evolutionary potential in quantitative characters under a balance between mutation and random genetic drift (Lande, 1995). Values less than 50 are required before drift causes the loss of SI alleles, which are under balancing selection (Byers and Meagher, 1992). For the two populations we examined, $N_{\mathrm{e}}$ was greater than 50 and less than 6000. We also estimated effective size, indirectly, for 11 additional populations in this species by multiplying their total census sizes by the average $N_{\mathrm{e}} / N$. Based on all 13 populations, $N_{\mathrm{e}}$ averaged 5357467 
$(\mathrm{SD}=4353949$; geometric mean $=21933.9)$ and ranged from 57.4 to 56476699.8. Four of the $13 \mathrm{H}$. herbacea populations had values lower than 5000 and six were smaller than 6000 , suggesting that some populations are at risk of losing neutral genetic variation. Only two populations were smaller than 50 indicating that drift may not be strong enough to influence the diversity of mating types within most populations. These crude predictions need to be tested using population surveys of neutral and ecological important diversity within natural populations.

Our study of $N_{\mathrm{e}}$ may have practical significance for the conservation and restoration of $H$. herbacea. The species is globally rare in both Canada and USA. Our estimates of effective size suggest that some populations may be small enough to cause the loss of genetic diversity, which in some cases may have ecological consequences. Through the elasticity analyses, our research also provides potential tools for management of these populations. Specifically, the stages of the life cycle that are most influential of effective size could be the focus of additional research to determine the underlying genetic and ecological factors limiting these stages (Schemske et al, 1994). The critical stages can also form the basis of more prescriptive management practices designed to alter population dynamics and reduce loss of diversity (Heppell et al, 2000). In $H$. herbacea, monitoring and augmenting the survival of nonsexual and sexual adults and clonal expansion may provide the most effective strategy to maintain and even increase population effective size in this species.

\section{Acknowledgements}

We thank Chris Hussell for field assistance, K Yonezawa, $\mathrm{J}$ Windus and T Waite for valuable discussions. Research was funded by an operating grant to $\mathrm{BCH}$ from the Natural Sciences and Engineering Research Council of Canada.

\section{References}

Balloux F, Lehmann L, de Meeûs T (2003). The population genetics of clonal and partially clonal diploids. Genetics 164: 1635-1644.

Basset P, Balloux F, Perrin N (2001). Testing demographic models of effective population size. Proc Roy Soc London B 268: 311-317.

Begon M, Chadburn R, Bishop JA, Keill C (1980). Genetic variation in a semi-natural Drosophila population after a bottleneck. I. Lethals, and their allelism and effective population size. Genetica 66: 11-20.

Bengtsson BO (2003). Genetic variation in organisms with sexual and asexual reproduction. J Evol Biol 16: 189-199.

Brown AHD, Allard RW (1970). Estimation of the mating system in open-pollinated maize populations using isozyme polymorphisms. Genetics 66: 133-145.

Byers DL, Meagher TR (1992). Mate availability in small populations of plant species with homomorphic sporophytic self-incompatibility. Heredity 68: 353-359.

Campbell LG (2001). Pollen Limitation in Small Populations of the Self-Incompatible Plant Hymenoxys herbacea. MSc Thesis, University of Guelph.

Campbell LG, Husband BC, Oldham M (2001). Status report on the Lakeside Daisy (Hymenoxys herbacea). Report to the Committee on the Status of Endangered Status of Wildlife in Canada.
Cusick AW (1991). Hymenoxys herbacea (Asteraceae): an endemic species of the Great Lakes region. Rhodora 93: 238-241.

Crow JF (1954). Breeding structure of populations. II. Effective population number. In: Kempthorn O, Bancroft TA, Gowen JW, Lush JL (eds) Statistics and Mathematics in Biology. Iowa State College Press: Ames, IA. pp 543-556.

de Kroon H, van Growenendael J, Ehrlèn J (2000). Elasticities: a review of methods and model limitations. Ecology 81: 607-618.

De Mauro MM (1989). Aspects of the Reproductive Biology of the Endangered Hymenoxys acaulis var. glabra: Implications for Conservation. MSc Thesis, University of Illinois.

De Mauro MM (1993). Relationship of breeding system to rarity in the Lakeside Daisy (Hymenoxys acaulis var. glabra). Conserv Biol 7: 542-550.

de Nettancourt D (1977). Incompatibility in Angiosperms. Springer-Verlag: Berlin, Heidelberg, New York.

Eckert CG, Barrett SCH (1994). Inbreeding depression in partially self-fertilizing Decodon verticillatus (Lythraceae): population-genetic and experimental analyses. Evolution 48: 952-964.

Ellstrand NC, Roose ML (1987). Patterns of genotypic diversity in clonal plant species. Am J Bot 74: 123-131.

Emigh TH, Pollak E (1979). Fixation probabilities and effective population numbers in diploid populations with overlapping generations. Theor Popul Biol 15: 86-107.

Frankham R (1995). Effective population size/adult population size ratios in wildlife: a review. Genet Res 66: 95-107.

Gabrielsen TM, Brochmann C (1998). Sex after all: high levels of diversity detected in the arctic clonal plant Saxifraga cernua using RAPD markers. Mol Ecol 7: 1701-1708.

Hall P, Walker S, Bawa K (1996). Effect of forest fragmentation on genetic diversity and mating system in a tropical tree, Pithecellobium elegans. Conserv Biol 10: 757-768.

Hebert PDN, Beaton MJ (1989). Methodologies for Allozyme Analysis using Cellulose Acetate Electrophoresis, A Practical Handbook. University of Windsor: Windsor, Canada.

Heppell SS, Crouse DT, Crowder LB (2000). Using matrix models to focus research and management efforts in conservation. In: Ferson S, Burgman M (eds) Quantitative Methods for Conservation Biology. Springer-Verlag: New York. pp 148-168.

Heywood JS (1986). The effect of plant size variation on genetic drift in populations of annuals. Am Nat 127: 851-861.

Hill WG (1972). Effective size of populations with overlapping generations. Theor Popul Biol 3: 278-289.

Hill WG (1979). A note on effective population size with overlapping generations. Genetics 92: 317-322.

Husband BC, Barrett SCH (1992). Genetic drift and the maintenance of the style length polymorphism in tristylous populations of Eichhornia paniculata (Pontederiaceae). Heredity 69: 440-449.

Jackson JBC, Buss LW, Cook RE (1985). Population Biology and Evolution of Clonal Organisms. Yale University Press: New Haven, Conn.

Kimura M, Crow JF (1963). The measurement of effective population number. Evolution 17: 279-288.

Lande R (1995). Mutation and conservation. Conserv Biol 9: 782-791.

Manchenko GP (1994). Handbook of Detection of Enzymes on Electrophoretic Gels. CRC Press: London.

Milgroom MG (1996). Recombination and the multilocus structure of fungal populations. Annu Rev Phytopathol 34: 457-477.

Nunney L (1991). The influence of age structure and fecundity on effective population size. Proc Roy Soc London B 246: 71-76.

Nunney L (1993). The influence of mating system and overlapping generations on effective population size. Evolution 47: 1329-1341.

Nunney L (1996). The influence of variation in female fecundity on effective population size: demography meets population genetics. TREE 8: 234-239. 
Olfelt JP, Furnier GR, Luby JJ (1998). Reproduction and development of the endangered Sedum integrifolium ssp. Leedyi (Crassulaceae). Am J Bot 85: 346-351.

Orive ME (1993). Effective population size in organisms with complex life-histories. Theor Popul Biol 44: 316-340.

Richman AD, Kohn JR (1999). Self-incompatibility alleles from Physalis: implications for historical inference from balanced genetic polymorphisms. Proc Natl Acad Sci USA 96: $168-172$.

Ritland K, Jain SK (1981). A model for the estimation of outcrossing rate and gene frequencies using $n$ independent loci. Heredity 47: 35-52.

Schemske DW, Husband BC, Ruckelshaus MH, Goodwilllie C, Parker IM, Bishop JG (1994). Evaluating approaches to the conservation of rare and endangered plants. Ecology 75: 584-606.

Van Groenendael JM, de Kroon H (1990). Clonal Growth in Plants: Regulation and Function. SPB Academic Publishing: The Hague, The Netherlands.
Vucetich JA, Waite TA (1998). Number of censuses required for demographic estimation of effective population size. Conserv Biol 12: 1023-1030.

Vucetich JA, Waite TA, Nunney L (1997). Fluctuating population size and the ratio of effective to census population size. Evolution 51: 2017-2021.

Wright S (1931). Evolution in Mendelian populations. Genetics 16: $97-159$

Wright S (1969). Evolution and the Genetics of Populations. II. The Theory of Gene Frequencies. University of Chicago Press: Chicago, IL.

Yonezawa K (1997). Effective population size of plant species propagating with a mixed sexual and asexual reproduction system. Genet Res 70: 251-258.

Yonezawa K, Kinoshita E, Watano Y, Zentoh H (2000). Formulation and estimation of the effective size of stagestructured populations in Fritillaria camtschatcensis, a perennial herb with a complex life history. Evolution 54: 2007-2013. 